\title{
Initial Value Determination of Chua System with Hidden Attractors and Its DSP Implementation
}

\author{
Xianming Wu $\mathbb{D},{ }^{1}$ Weijie Tan $\mathbb{D}^{2}$ and Huihai Wang $\mathbb{D}^{3}$ \\ ${ }^{1}$ School of Mechanical \& Electrical Engineering, Guizhou Normal University, Guiyang 550025, China \\ ${ }^{2}$ State Key Laboratory of Public Big Data, Guizhou University, Guiyang 550025, China \\ ${ }^{3}$ School of Physics and Electronics, Central South University, Changsha 410083, China \\ Correspondence should be addressed to Huihai Wang; wanghuihai_csu@csu.edu.cn
}

Received 7 May 2020; Accepted 20 June 2020; Published 24 July 2020

Guest Editor: Chun-Lai Li

Copyright ( $\odot 2020$ Xianming Wu et al. This is an open access article distributed under the Creative Commons Attribution License, which permits unrestricted use, distribution, and reproduction in any medium, provided the original work is properly cited.

In this paper, a method for determining the initial value of the hidden attractors in the Chua system is studied. The initial value of the hidden attractors can be calculated quickly and accurately by the proposed method, and the hidden attractors can be found by numerical simulation. Then, the initial values of the hidden attractors are set accurately by digital signal processor (DSP), so as to the circuit realization of the chaotic system with hidden attractors is performed. The results show that the numerical simulation results of Matlab are consistent with the experimental results of DSP.

\section{Introduction}

In the last three decades, chaos has been widely used in neural networks [1-5], electronic circuits [6], image processing [7-10], random number generators [11], system synchronization [12-14], and secure communication [15-18] because of its characteristics of aperiodic, continuous broadband, noise-like, and unpredictable for a long time. Since Lorenz puts forward the first chaotic system in the study of atmospheric motion [19], the research and exploration of the chaotic system composed of ordinary differential equations have attracted researchers' great attention, and many new chaotic systems with complex dynamic attractors, such as multiscroll attractors [20] and coexistence attractors [21-23] have been constantly produced.

Because the domain of attraction of the hidden attractor does not intersect with any small neighbourhood of the equilibrium point, there is no general method to predict the existence of the hidden attractor, so it is of great theoretical and practical significance to study the hidden attractor in the field of machinery and so on [24, 25]. In 2011, Leonov et al. proposed a locating algorithm for hidden attractors [26] and used the algorithm to find hidden attractors of the Chua system. Since then, the research on hidden attractors has attracted extensive interest of scholars. In 2012, Leonov et al. used the algorithm to find the hidden attractor in the Chua system with hyperbolic tangent function as nonlinear function [27]. In 2014, Zhao et al. used the algorithm to find the hidden attractor in a generalized autonomous PolDuffing system [28]. In the same year, Li et al. found the twin hidden attractors in the Chua system [29]. In 2017, Zhao et al. found the hidden attractor in a modified Chua system [30]. In the same year, Kuznetsov et al. also found coexistence limit cycle and symmetric hidden attractors in the Chua system [31]. Stankevich et al. analysed the scenario of the birth of the hidden attractor from its attractor basin in the Chua system [32]. These attractors are different from the classical Lorenz attractors, Chua attractors, and Chen attractors. They are not near the equilibrium point and cannot be calculated by traditional methods. Leonov et al. proposed an algorithm to determine the initial value of the hidden attractors and found the hidden attractors.

In 2016, Bao et al. designed the chaotic circuit of the chaotic system and found the hidden attractor of the system by PSIM simulation [33]. In [34], a three-dimensional autonomous chaotic circuit is designed, and the hidden attractor of the system is found by PSpice simulation. In 
[35], a 5-D memristor chaotic circuit is designed. The hidden multiscroll attractors and hidden multiwing attractors are found by PSpice simulation. In [36], a 5D extreme multistable chaotic circuit is designed, and the hidden attractor of the system is found by PSpice simulation. In [37], a fourdimensional chaotic circuit is designed, and the coexistence hidden attractor of the system is found by PSpice simulation. In [38], a new chaotic circuit is designed by introducing the cosh function into the system in [37], and the coexistence hidden attractor of the system is found by PSpice simulation. In [39], by introducing a memristor to improve Fitzhugh-Nagumo circuit, a new memristor chaotic circuit is designed, and the hidden attractor is found by PSIM simulation. However, the hidden attractor is studied by circuit simulation software, but the initial state of the experimental circuit is random, so the initial value of the hidden attractor cannot be set accurately.

In this paper, we study the method to determine the initial value of the hidden attractors in the Chua system. Its initial value of the hidden attractors can be set accurately by DSP, and the circuit realization of the chaotic system with hidden attractors is performed. The results show that the numerical simulation results of Matlab are consistent with the experimental results of DSP.

The rest of this work is organized as follows. Section 2 describes initial value determining algorithm for the Chua system with hidden attractors. Section 3 calculates initial values of hidden attractors and finds its hidden attractors. The Chua system with hidden attractors is implemented by DSP in Section 4. Finally, we conclude in Section 5.

\section{Initial Value Determining Algorithm for Hidden Attractors}

According to the initial value determining algorithm for the chaotic system with hidden attractors in [26], the Chua system with hidden attractors is

$$
\left\{\begin{array}{l}
\frac{\mathrm{d} x}{\mathrm{~d} t}=a(y-x)-a f(x) \\
\frac{\mathrm{d} y}{\mathrm{~d} t}=x-y+z \\
\frac{\mathrm{d} z}{\mathrm{~d} t}=-b y-c z
\end{array}\right.
$$

where $f(x)=m x+0.5(n-m)(|x+1|-|x-1|), x, y$, and $z$ are system variables, and $a, b, c, m$, and $n$ are system constants.

Now, system (1) is rewritten as lure system:

$$
\frac{\mathrm{d} x}{\mathrm{~d} t}=P x+q \psi\left(r^{*} x\right) \quad, x \in R^{3},
$$

where $P=\left(\begin{array}{ccc}-a(1+m) & a & 0 \\ 1 & -1 & 1 \\ 0 & -b & -c\end{array}\right), q=\left(\begin{array}{c}-a \\ 0 \\ 0\end{array}\right), r=\left(\begin{array}{l}1 \\ 0 \\ 0\end{array}\right)$, and $\psi(\sigma)=0.5(n-m)(|\sigma+1|-|\sigma-1|)$.

Let $k$ be the coefficient of harmonic linearization, and $\varepsilon$ be an infinitesimal number, and equation (2) can be rewritten as

$$
\frac{\mathrm{d} x}{\mathrm{~d} t}=P_{0} x+q \varepsilon \delta\left(r^{*} x\right)
$$

where $\quad P_{0}=\left(\begin{array}{ccc}-a(1+m+k) & a & 0 \\ 1 & -1 & 1 \\ 0 & -b & -c\end{array}\right), \quad \lambda_{1,2}^{P_{0}}= \pm i \omega_{0}$, $\lambda_{3}^{P_{0}}=-d<0$, and $\delta(\sigma)=\psi(\sigma)-k \sigma$.

Using nonsingular linear transformation $x=S y$, equation (3) can be transformed as

$$
\frac{\mathrm{d} y}{\mathrm{~d} t}=H x+e \varepsilon \phi\left(u^{*} y\right)
$$

where $H=\left(\begin{array}{ccc}0 & -\omega_{0} & 0 \\ \omega_{0} & 0 & 0 \\ 0 & 0 & -d\end{array}\right), e=\left(\begin{array}{c}e_{1} \\ e_{2} \\ 1\end{array}\right)$, and $u=\left(\begin{array}{c}1 \\ 0 \\ -h\end{array}\right)$.

The transfer function of equation (4) can be expressed as

$$
W_{H}(p)=\frac{-e_{1} p+e_{2} \omega_{0}}{p^{2}+\omega_{0}^{2}}+\frac{h}{p+d}
$$

The transfer functions of system (3) can be expressed as

$$
W_{P_{0}}(p)=r^{*}\left(P_{0}-p I\right)^{-1} q,
$$

where $p$ is complex variables, $\omega_{0}$ is the initial frequency, which can be calculated by $\operatorname{ImWH}\left(\omega_{0}\right) \mathrm{F} 0$, and $k$ is the harmonic linearization coefficient, which can be calculated by $k=-\left(\operatorname{Re} W_{H} i \omega_{0}\right)^{-1}$. From the equivalence of the transfer functions of systems (3) and (4), it can be concluded:

$$
\left\{\begin{array}{l}
k=\left(\frac{-a(m+m c+c)+\left(\omega_{0}^{2}-b-c\right)}{a(1+c)}\right) \\
d=\left(\frac{\omega_{0}^{2}-b+1+a+c+c^{2}}{(1+c)}\right) \\
h=\frac{a\left(c+b-(1+c) d+d^{2}\right)}{\left(\omega_{0}^{2}+d^{2}\right)} \\
e_{1}=\frac{a\left(c+b-(1+c) d-\omega_{0}^{2}\right)}{\left(\omega_{0}^{2}+d^{2}\right)} \\
e_{2}=\frac{a\left((1+c-d) \omega_{0}^{2}+d(c+b)\right)}{\left(\omega_{0}\left(\omega_{0}^{2}+d^{2}\right)\right)}
\end{array}\right.
$$

System (3) is transformed by nonsingular linear transformation, and it can be concluded:

$$
\left\{\begin{array}{l}
H=S^{-1} P_{0} S \\
e=S^{-1} q \\
u^{*}=r^{*} S
\end{array}\right.
$$

Let $S=\left(\begin{array}{lll}s_{11} & s_{12} & s_{13} \\ s_{21} & s_{22} & s_{23} \\ s_{31} & s_{32} & s_{33}\end{array}\right)$. We can obtain $s_{11}=1, s_{12}=0$, $s_{13}=-h, s_{21}=m+k+1, s_{22}=-\left(\omega_{0} / a\right), s_{23}=-(h(a(m+$ $k+1)-d)) / a, \quad s_{31}=\left(a(m+k)-\omega_{0}^{2}\right) / a, \quad s_{32}=-(a(c+b)$ $\left.(m+k)+a b-c \omega_{0}^{2}\right) / a \omega_{0}$, and $s_{33}=h((a(m+k)(d-1)+$ $d(1+a-d)) / a)$. 
For the infinitesimal number $\varepsilon$, the initial value of (4) is

$$
y(0)=\left(\begin{array}{l}
y_{1}(0) \\
y_{2}(0) \\
y_{3}(0)
\end{array}\right)=\left(\begin{array}{c}
g_{0} \\
0 \\
0
\end{array}\right) .
$$

From equation (9), the relationship between the initial values of equations (3) and (4) can be obtained:

$$
x(0)=S y(0)=S\left(\begin{array}{l}
x_{1}(0) \\
x_{2}(0) \\
x_{3}(0)
\end{array}\right)=\left(\begin{array}{l}
g_{0} s_{11} \\
g_{0} s_{21} \\
g_{0} s_{31}
\end{array}\right) .
$$

In this way, the initial value of system (1) is

$$
\begin{aligned}
& x(0)=g_{0}, \\
& y(0)=-g_{0}(m+k+1), \\
& z(0)=g_{0} \frac{a(m+k)-\omega_{0}^{2}}{a},
\end{aligned}
$$

where the description function of $\mathrm{a}_{0}$ can be calculated as

$$
\begin{aligned}
\Phi(g)= & \int_{0}^{2 \pi / \omega_{0}}\left[\delta_{1}\left(\left(\cos \omega_{0} t\right) g,\left(\sin \omega_{0} t\right) g, 0\right) \cos \omega_{0} t\right. \\
& \left.+\delta_{2}\left(\left(\cos \omega_{0} t\right) g,\left(\sin \omega_{0} t\right) g, 0\right) \sin \omega_{0} t\right] \mathrm{d} t
\end{aligned}
$$

and the description function satisfies $\Phi\left(g_{0}\right)=0$ and $\left.b_{1}(d \Phi(g) / d g)\right|_{g=g_{0}} \neq 0$.

\section{Numerical Simulation}

According to the algorithm in [26], the initial values are $x_{0}=[5.9,0.3720,-8.4291]$ and $x_{00}=[-5.9,-0.3720,8.4291]$. Based on the calculated initial value, the phase diagram is shown in Figure 1, and the attractor basin is shown in Figure 2.

From Figure 1, it can be seen that the hidden attractor can be found according to the initial value calculated by the algorithm in [26]. From Figure 2, the blue centre region is stable equilibrium, the red region is period-1 limit cycle, and the cyan region is divergent.

\section{DSP Implementation of Chua System with Hidden Attractors}

The realization of the chaotic system by hardware circuit is the most common method to verify the design of new chaotic system, including analog circuit and digital circuit. Analog circuit mainly adopts discrete components [13] and integrates circuit (IC) [40-42] design method, while digital circuit mainly adopts FPGA [43] and DSP [44-46]. It is difficult to design and debug chaotic circuit with discrete components, and the circuit is bulky. When using IC to design the chaotic oscillator, the chip area is greatly reduced, but the design of IC requires high chip technology, and the number of wings or scrolls of the attractor is difficult to control. Because the analog circuit cannot accurately set the initial state of the system and cannot reach the calculated initial value of the hidden attractors. FPGA and DSP have high-speed data processing capability and can realize various processing algorithms through software programming $[47,48]$, which can conveniently realize the nonlinear characteristics of the chaotic system with hidden attractors.

4.1. Implementation of the Chaotic System. In this part, the chaotic system with hidden attractors is implemented on DSP platform. The block diagram of the working principle is shown in Figure 3. In the experiments, the Texas Instrument DSP device TMS320F28335 is employed. It is a 32 bit DSP running at $150 \mathrm{MHz}$ with floating point operations. Such a high-speed clock rate is considered to be sufficient. In order to observe the phase diagrams of the attractor on the oscilloscope, the digital chaotic sequences generated on DSP are converted into analog signals. DAC8552, a 16 bit digitalto-analog converter with dual channels, is adopted. It connects DSP through SPI (serial peripheral interface).

The flowchart of the programming is shown in Figure 4. In the program, in order to reduce the effect of finite computing precision in digital circuits, all data types are defined as long double. After initializing DSP, we set the initial conditions, including initial values of state variables, and the system parameters. Iterative computation is started according to the initial values $\left[x_{0}, y_{0}, z_{0}\right]$. To keep the iteration not being affected by data processing, it is necessary to push the results of each iteration into the stack. Data processing includes two steps. Firstly, an appropriate positive number is added to all data to make sure all data is greater than zero. Secondly, all data is rescaled and truncated to make the output adapting the 16 bit digital-to-analog converter.

4.2. Runge-Kutta4 (RK4) Algorithm. According to the required iterative equation in Figure 4, we use the RK4 algorithm to realize the iterative equation of the Chua system with hidden attractors. RK4 is a derivative of Runge-Kutta basic model, which is used to solve ordinary differential equations with high accuracy, and mostly has proved itself superior to other solutions. RK4 algorithm is expressed as

$$
\left\{\begin{array}{l}
y_{i+1}=y_{i}+\frac{h}{6}\left(k_{1}+2 k_{2}+2 k_{3}+k_{4}\right) \\
k_{1}=f\left(x_{i}, y_{i}\right) \\
k_{2}=f\left(x_{i}+\frac{h}{2}, y_{i}+\frac{h}{2} k_{1}\right) \\
k_{3}=f\left(x_{i}+\frac{h}{2}, y_{i}+\frac{h}{2} k_{2}\right) \\
k_{4}=f\left(x_{i}+h, y_{i}+h k_{3}\right)
\end{array}\right.
$$

According to the values of $h, x_{i}$, and $y_{i}$ and calculating the values of $k_{1}, k_{2}, k_{3}$, and $k_{4}$, we can attain the value of $y_{i+1}$. Three equations of system (1) are substituted into equation (13), and the three state variables $(x, y, z)$ of system (1) are solved, respectively: 

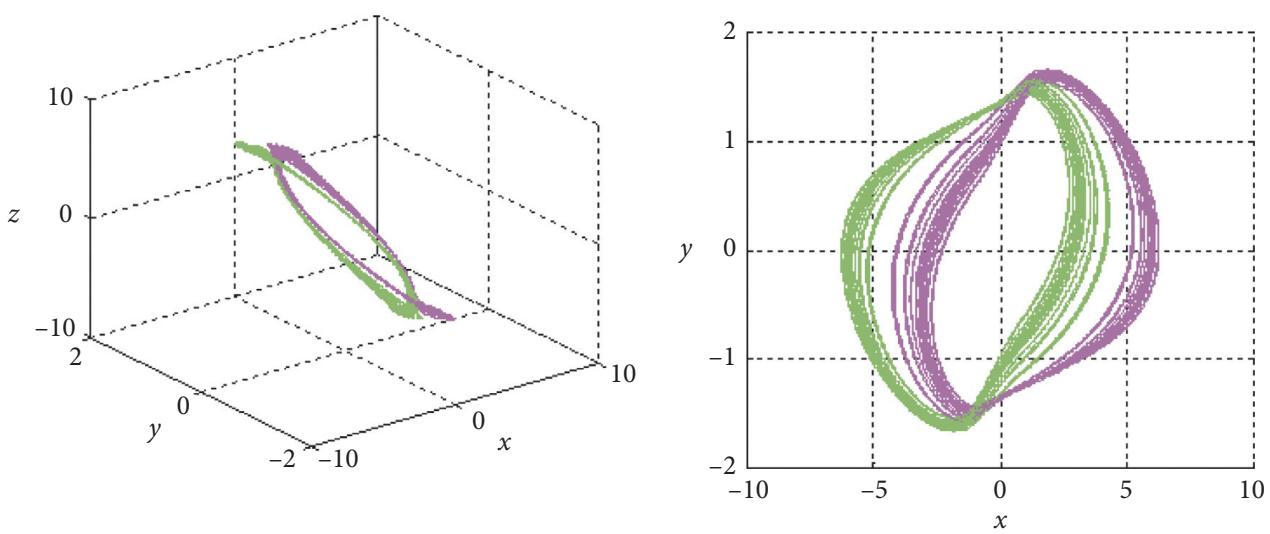

(a)



(c) (b)

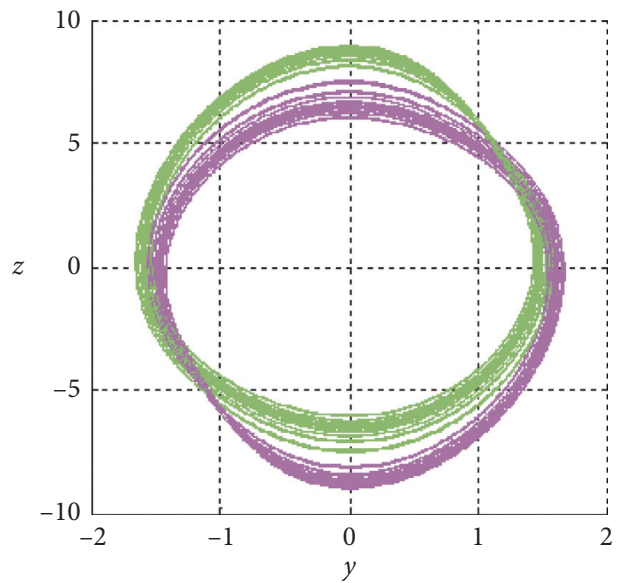

(d)

Figure 1: Phase diagrams of system (1); initial values: (5.9 $0.3720-8.4291)$ (magenta) and (-5.9 -0.3720 8.4291) (green).

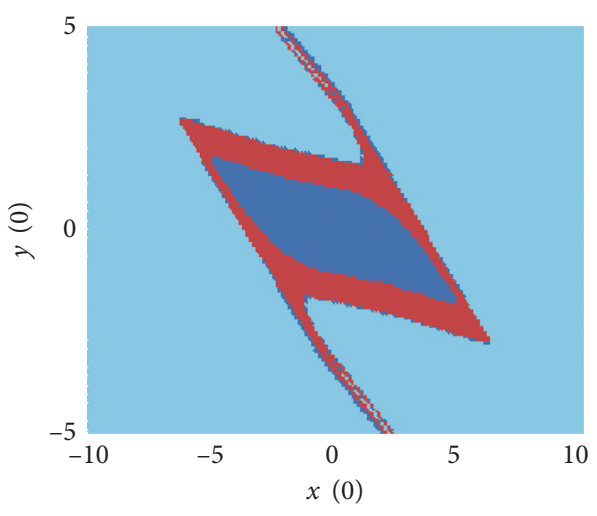

(a)

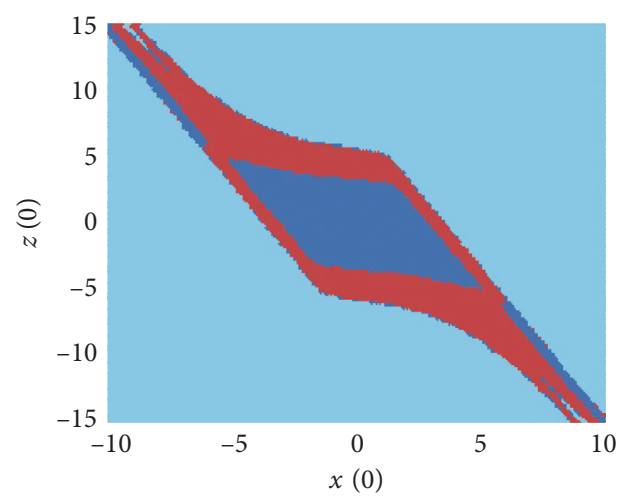

(b)

Figure 2: Continued. 


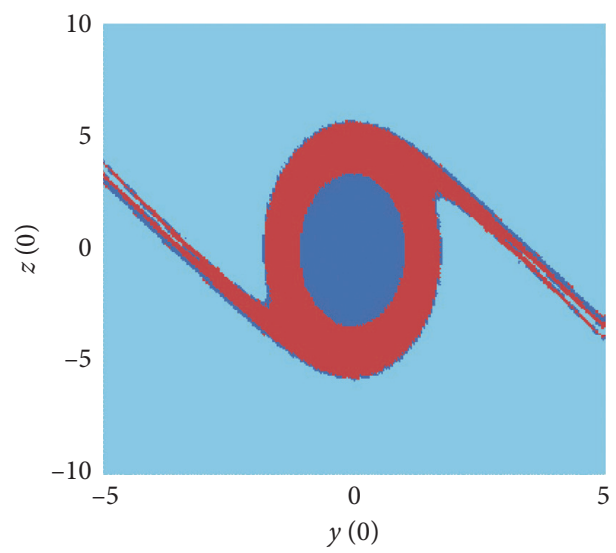

(c)

Figure 2: Attractor basin of system (1): (a) initial value $x-y$ plane; (b) initial value $x-z$ plane; (c) initial value $y-z$ plane.

\begin{tabular}{|c|c|c|c|}
\hline $\begin{array}{c}\text { DSP } \\
(\mathrm{TMS} 320 \mathrm{~F} 28335)\end{array}$ & SPI & $\mathrm{D}$ & $\longrightarrow \begin{array}{c}\text { Oscilloscope } \\
\text { (Tektronix MDO3104) }\end{array}$ \\
\hline
\end{tabular}

FIGURE 3: Working principle for implementing the Chua system with hidden attractors on DSP.

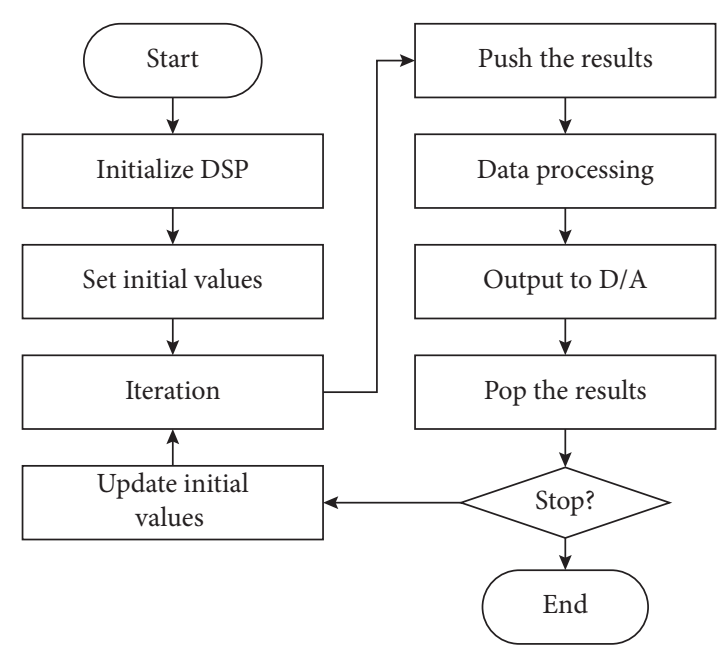

FIGURE 4: Flowchart for DSP implementation of the Chua system with hidden attractors.

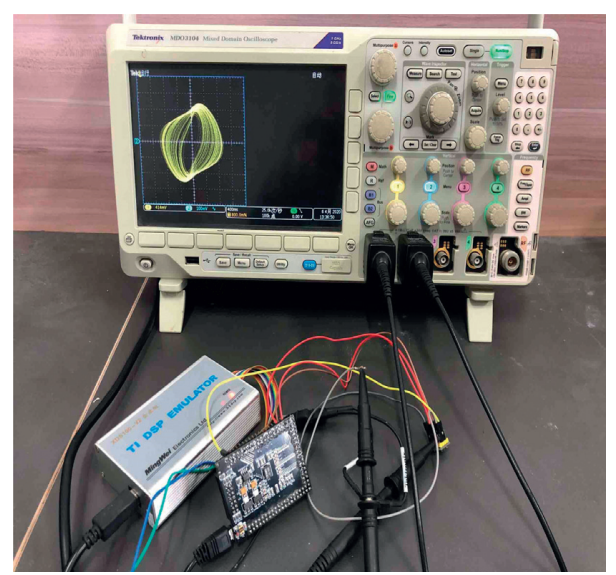

Figure 5: Hardware part of DSP implementation. 




(a)

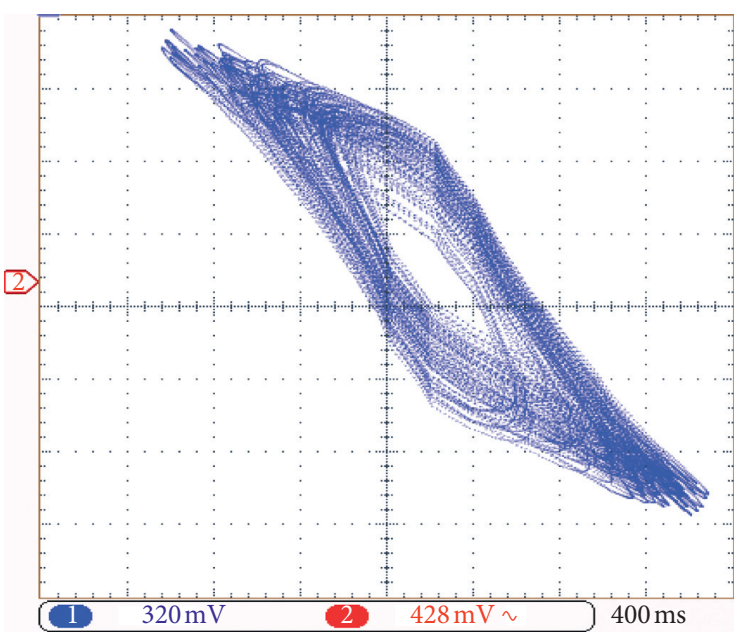

(b)

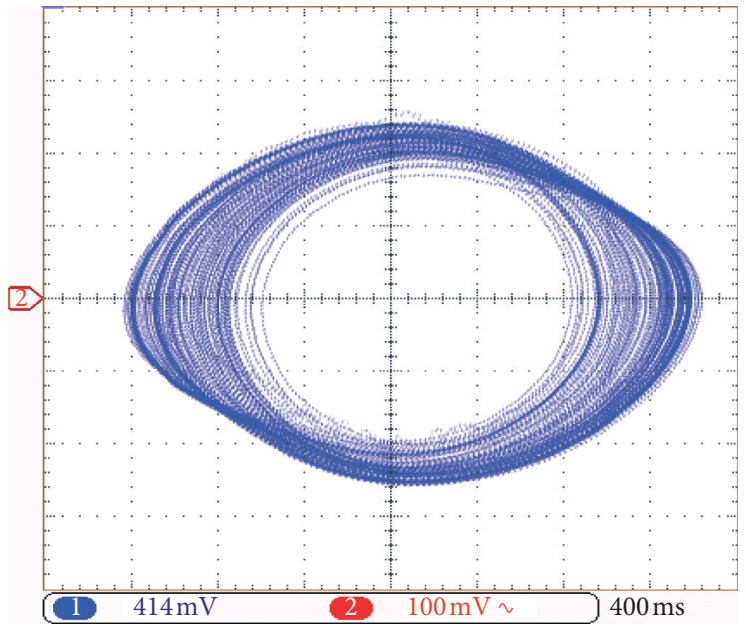

(c)

FIgURE 6: Hidden attractors by DSP implementation for $a=8.4562 ; b=12.0732 ; c=0.0052 ; m=-1.1468 ; n=-0.1768$ : (a) $x-y$ plane; (b) $x-z$ plane; (c) $y$ - $z$ plane.

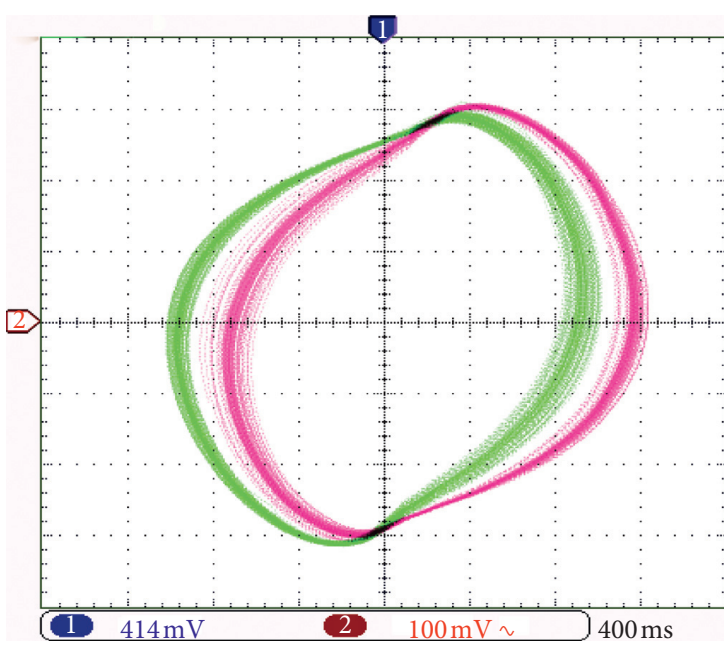

(a)

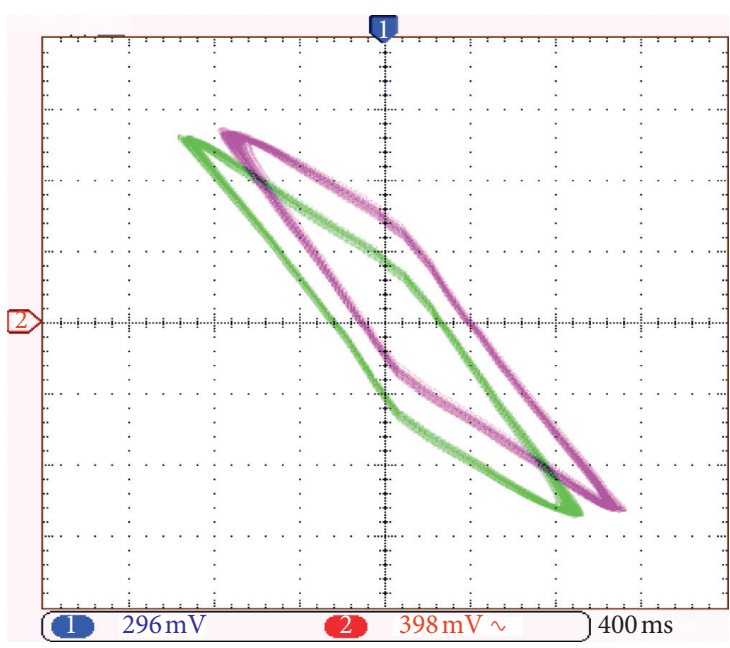

(b)

Figure 7: Continued. 


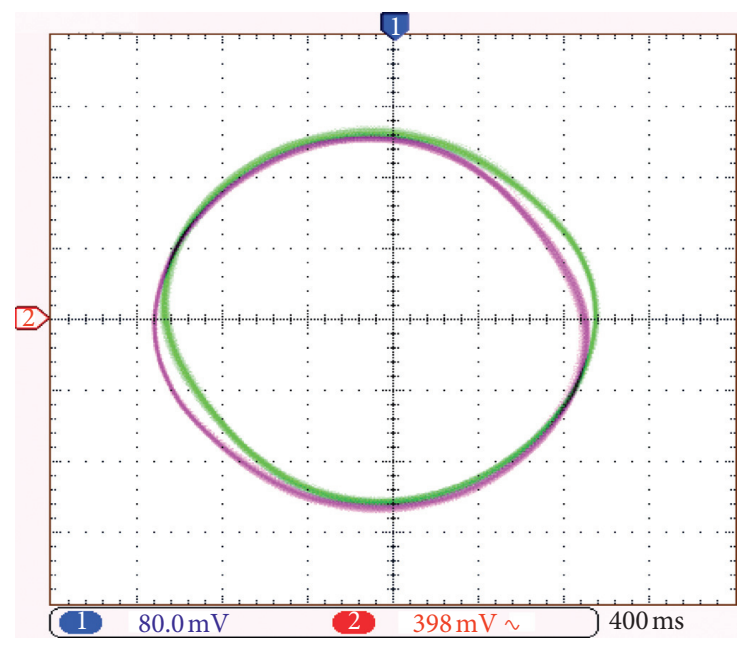

(c)

FIGURE 7: Hidden attractors by DSP implementation; initial values: (5.9 0.3720-8.4291) (magenta) and (-5.9 -0.3720 8.4291) (green): (a) $x$ - $y$ plane; (b) $x-z$ plane; (c) $y-z$ plane.

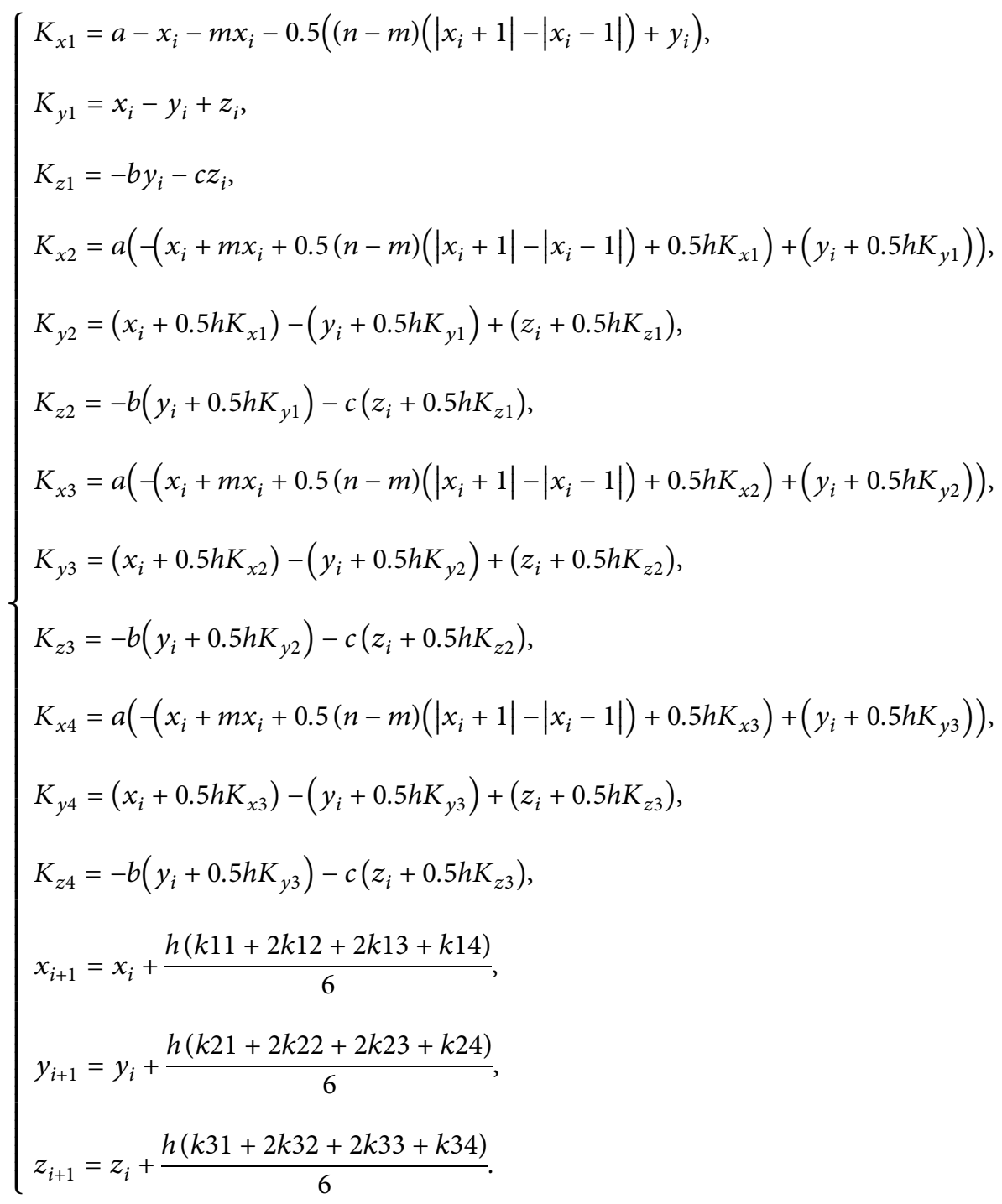


4.3. Circuit Implementation Using DSP. We set $h=0.001$, initial values $x_{0}=\left[\begin{array}{ll}5.9 & 0.3720-8.4291\end{array}\right]$ and $x_{00}=\left[\begin{array}{ll}5.9 & 0.3720\end{array}\right.$ 8.4291], when $a=8.4562, \quad b=12.0732, \quad c=0.0052$, $m=-1.1468$, and $n=-0.1768$. The system is realized by the DSP platform. Figure 5 shows the hardware part of DSP. Phase diagrams of the system are captured randomly by the oscilloscope, as shown in Figure 6. When $a=8.4, b=12.1$, $c=0.005, m=-1.1$, and $n=0.1$, its phase diagrams is shown in Figure 7. It indicates that the Chua system with hidden attractors is realized successfully on the DSP platform.

From Figures 5-7, it is observed that the DSP circuit can generate two hidden attractors.

\section{Conclusions}

In this paper, we calculate the initial values of the Chua system with hidden attractors, find its hidden attractors, and obtain its phase diagram and attractor basin. Since the analog circuit cannot accurately set its initial state and cannot achieve the calculated initial value of the hidden attractor, this paper uses DSP to realize the chaos system with hidden attractors. The results show that the numerical simulation is consistent with the experimental results of DSP, which provides a practical method for the circuit implementation of the chaotic system with hidden attractors. In the next work, we will study the hidden attractor applied to secure communication.

\section{Data Availability}

The data used to support the findings of this study are included within the article.

\section{Conflicts of Interest}

The authors declare that they have no conflicts of interest.

\section{Authors' Contributions}

The work presented in this paper was a collaboration of all authors. Xianming WU contributed the idea and wrote the paper. Weijie Tan and Huihai Wang did the simulation analysis and reviewed the paper.

\section{Acknowledgments}

This work was supported by the National Natural Science Foundation of China (no. 61741104), Science and Technology Foundation of Guizhou Province of China (no. [2018]1115), Science and Technology Plan Project of Guizhou Province of China (no. [2018]5769), and Doctoral Scientific Research Foundation of Guizhou Normal University (2017).

\section{References}

[1] Z. T. Njitacke, S. D. Isaac, J. Kengne, A. N. Negou, and G. D. Leutcho, "Extremely rich dynamics from hyperchaotic hopfield neural network: hysteretic dynamics, parallel bifurcation branches, coexistence of multiple stable states and its analog circuit implementation," The European Physical Journal Special Topics, vol. 229, no. 6-7, pp. 1133-1154, 2020.

[2] F. Yu, L. Liu, L. Xiao, K. Li, and S. Cai, "A robust and fixedtime zeroing neural dynamics for computing time-variant nonlinear equation using a novel nonlinear activation function," Neurocomputing, vol. 350, pp. 108-116, 2019.

[3] F. Wang, L. Zhang, S. Zhou, and Y. Huang, "Neural networkbased finite-time control of quantized stochastic nonlinear systems," Neurocomputing, vol. 362, pp. 195-202, 2019.

[4] J. Jin, L. Zhao, M. Li, F. Yu, and Z. Xi, "Improved zeroing neural networks for finite time solving nonlinear equations," Neural Computing and Applications, vol. 32, no. 9, pp. 4151-4160, 2020.

[5] H. Lin and C. Wang, "Influences of electromagnetic radiation distribution on chaotic dynamics of a neural network," Applied Mathematics and Computation, vol. 369, Article ID 124840, 2020.

[6] Y. M. Tan and C. H. Wang, "A simple locally active memristor and its application in HR neurons," Chaos, vol. 30, no. 5, Article ID 53118, 2020.

[7] L. M. Zhang, K. H. Sun, and W. H. Liu, "A novel color image encryption scheme using fractional-order hyperchaotic system and DNA sequence operations," Chinese Physics B, vol. 26, Article ID 100504, 2017.

[8] G. Cheng, C. Wang, and H. Chen, "A novel color image encryption algorithm based on hyperchaotic system and permutation-diffusion architecture," International Journal of Bifurcation and Chaos, vol. 29, no. 9, Article ID 1950115, 2019.

[9] M. Zhou and C. Wang, "A novel image encryption scheme based on conservative hyperchaotic system and closed-loop diffusion between blocks," Signal Processing, vol. 171, Article ID 107484, 2020

[10] Z. Hua, Y. Zhou, and B. Bao, "Two-dimensional sine chaotification system with hardware implementation," IEEE Transactions on Industrial Informatics, vol. 16, no. 2, pp. 887-897, 2020.

[11] F. Yu, L. Liu, and S. Qian, "Chaos-based application of a novel multistable 5D memristive hyperchaotic system with coexisting multiple attractors," Complexity, vol. 2020, Article ID 8034196, 19 pages, 2020.

[12] L. Zhou, F. Tan, F. Yu, and W. Liu, "Cluster synchronization of two-layer nonlinearly coupled multiplex networks with multi-links and time-delays," Neurocomputing, vol. 359, no. 24, pp. 264-275, 2019.

[13] F. Yu, S. Qian, and X. Chen, “A new 4D four-wing memristive hyperchaotic system: dynamical analysis, electronic circuit design, shape synchronization and secure communication," International Journal of Bifurcation and Chaos, 2020.

[14] L. Zhou, F. Tan, and F. Yu, "A robust synchronization-based chaotic secure communication scheme with double-layered and multiple hybrid networks," IEEE Systems Journal, vol. 14, no. 2, pp. 2508-2519, 2020.

[15] Z. Hua, Y. Zhang, and Y. Zhou, "Two-dimensional modular chaotification system for improving chaos complexity," IEEE Transactions on Signal Processing, vol. 68, pp. 1937-1949, 2020.

[16] Z. Hua and Y. Zhou, "Exponential chaotic model for generating robust chaos," IEEE Transactions on Systems, Man, and Cybernetics: Systems, vol. 99, pp. 1-12, 2019.

[17] F. Yu, Z. Zhang, and L. Liu, "Secure communication scheme based on a new 5D multistable four-wing memristive hyperchaotic system with disturbance inputs," Complexity, vol. 2020, Article ID 5859273, 16 pages, 2020. 
[18] F. Kuang, S. Zhang, Z. Jin, and W. Xu, "A novel SVM by combining kernel principal component analysis and improved chaotic particle swarm optimization for intrusion detection," Soft Computing, vol. 19, no. 5, pp. 1187-1199, 2015.

[19] E. N. Lorenz, "Deterministic nonperiodic flow," Journal of the Atmospheric Sciences, vol. 20, no. 2, pp. 130-141, 1963.

[20] Q. Deng and C. Wang, "Multi-scroll hidden attractors with two stable equilibrium points," Chaos, vol. 29, no. 9, Article ID 93112, 2019.

[21] G. D. Leutcho, J. Kengne, and R. Kengne, "Remerging Feigenbaum trees, and multiple coexisting bifurcations in a novel hybrid diode-based hyperjerk circuit with offset boosting," International Journal of Dynamics and Control, vol. 7, no. 1, pp. 61-82, 2019.

[22] G. D. Leutcho and J. Kengne, "A unique chaotic snap system with a smoothly adjustable symmetry and nonlinearity: chaos, offset-boosting, antimonotonicity, and coexisting multiple attractors," Chaos, Solitons \& Fractals, vol. 113, pp. 275-293, 2018.

[23] G. D. Leutcho, J. Kengne, and T. FonzinFozin, "Multistability control of space magnetization in hyperjerk oscillator: a case study," Journal of Computational and Nonlinear Dynamics, vol. 15, no. 5, Article ID ID051004, 2020.

[24] L. Cui, M. Lu, and Q. Ou, "Analysis and circuit implementation of fractional order multi-wing hidden attractors," Chaos, Solitons \& Fractals, vol. 138, Article ID 109894, 2020.

[25] H. Lin, C. Wang, and Y. Tan, "Hidden extreme multistability with hyperchaos and transient chaos in a Hopfield neural network affected by electromagnetic radiation," Nonlinear Dynamics, vol. 99, no. 3, pp. 2369-2386, 2020.

[26] G. A. Leonov, N. V. Kuznetsov, and V. I. Vagaitsev, "Localization of hidden Chua's attractors," Physics Letters A, vol. 375, no. 23, pp. 2230-2233, 2011.

[27] G. A. Leonov, N. V. Kuznetsov, and V. I. Vagaitsev, "Hidden attractor in smooth Chua systems," Physica D: Nonlinear Phenomena, vol. 241, no. 18, pp. 1482-1486, 2012.

[28] H. T. Zhao, Y. P. Lin, and Y. X. Dai, "Hidden attractors and dynamics of a general autonomous van der Pol-Duffing oscillator," International Journal of Bifurcation and Chaos, vol. 24, no. 6, Article ID 1450080, 2014.

[29] Q. D. Li, H. Z. Zeng, and X. S. Yang, "On hidden twin attractors and bifurcation in the Chua's circuit," Nonlinear Dynamics, vol. 77, no. 1-2, pp. 255-266, 2014.

[30] H. Zhao, Y. Lin, and Y. Dai, "Hopf bifurcation and hidden attractor of a modified Chua's equation," Nonlinear Dynamics, vol. 90, no. 3, pp. 2013-2021, 2017.

[31] N. V. Kuznetsov, O. A. Kuznetsova, G. A. Leonov, T. N. Mokaev, and N. V. Stankevich, "Hidden attractors localization in Chua circuit via the describing function method," IFAC-PapersOnLine, vol. 50, no. 1, pp. 2651-2656, 2017.

[32] N. V. Stankevich, N. V. Kuznetsov, and G. A. Leonov, "Scenario of the birth of hidden attractors in the Chua circuit," International Journal of Bifurcation and Chaos, vol. 27, no. 12, Article ID 1730038, 2017.

[33] B.-C. Bao, M. Chen, Q. Xu, and P. Jiang, "Hidden attractors in a practical Chua's circuit based on a modified Chua's diode," Electronics Letters, vol. 52, no. 1, pp. 23-25, 2016.

[34] S. T. Kingni, S. Jafari, V.-T. Pham, and P. Woafo, "Constructing and analyzing of a unique three-dimensional chaotic autonomous system exhibiting three families of hidden attractors," Mathematics and Computers in Simulation, vol. 132, pp. 172-182, 2017.
[35] X. Y. Hu, C. X. Liu, and L. Liu, "Multi-scroll hidden attractors and multi-wing hidden attractors in a 5-dimensional memristive system," Chinese Physics B, vol. 26, no. 11, Article ID $110502,2017$.

[36] S. Jafari, A. Ahmadi, A. J. M. Khalaf, H. R. Abdolmohammadi, V.-T. Pham, and F. E. Alsaadi, "A new hidden chaotic attractor with extreme multi-stability," AEU-International Journal of Electronics and Communications, vol. 89, pp. 131135, 2018

[37] J. P. Singh and B. K. Roy, "Multistability and hidden chaotic attractors in a new simple 4-D chaotic system with chaotic 2torus behaviour," International Journal of Dynamics and Control, vol. 6, no. 2, pp. 529-538, 2018.

[38] V. R. F. Signing and J. Kengne, "Coexistence of hidden attractors, 2-torus and 3-torus in a new simple 4-D chaotic system with hyperbolic cosine nonlinearity," International Journal of Dynamics and Control, vol. 6, no. 4, pp. 1421-1428, 2018.

[39] H. Bao, W. Liu, and M. Chen, "Hidden extreme multistability and dimensionality reduction analysis for an improved nonautonomous memristive FitzHugh-Nagumo circuit," Nonlinear Dynamics, vol. 96, no. 3, pp. 1879-1894, 2019.

[40] J. Jin, "Programmable multi-direction fully integrated chaotic oscillator," Microelectronics Journal, vol. 75, pp. 27-34, 2018.

[41] J. Jin and L. Zhao, "Low voltage low power fully integrated chaos generator," Journal of Circuits, Systems and Computers, vol. 27, no. 10, Article ID 1850155, 2018.

[42] J. Jin and L. Cui, "Fully integrated memristor and its application on the scroll-controllable hyperchaotic system," Complexity, vol. 2019, Article ID 4106398, 8 pages, 2019.

[43] F. Yu, H. Shen, and L. Liu, "CCII and FPGA realization: a multistable modified four-order autonomous Chua's chaotic system with coexisting multiple attractors," Complexity, vol. 2020, Article ID 5212601, 17 pages, 2020.

[44] Y. Xiao, K. Sun, and S. He, "Constructing chaotic map with multi-cavity," The European Physical Journal Plus, vol. 135, no. 1, p. 21, 2020.

[45] H. H. Wang, K. H. Sun, and S. B. He, "Dynamic analysis and implementation of a digital signal processor of a fractionalorder Lorenz-Stenflo system based on the adomian decomposition method," Physica Scripta, vol. 90, no. 1, Article ID 15206, 2014.

[46] W. Ai, K. Sun, and Y. Fu, "Design of multiwing-multiscroll grid compound chaotic system and its circuit implementation," International Journal of Modern Physics C, vol. 29, no. 6, Article ID 1850049, 2018.

[47] J.-L. Zhang, W.-Z. Wang, X.-W. Wang, and Z.-H. Xia, "Enhancing security of FPGA-based embedded systems with combinational logic binding," Journal of Computer Science and Technology, vol. 32, no. 2, pp. 329-339, 2017.

[48] V. Guglielmi, P. Pinel, D. Fournier-Prunaret, and A.-K. Taha, "Chaos-based cryptosystem on DSP," Chaos, Solitons \& Fractals, vol. 42, no. 4, pp. 2135-2144, 2009. 